\title{
Downregulation of the Spi-1/PU.1 oncogene induces the expression of TRIM10/HERF1, a key factor required for terminal erythroid cell differentiation and survival
}

\author{
Rand Blaybel $^{1}$, Orianne Théoleyre ${ }^{1}$, Alexandre Douablin ${ }^{1}$, Faouzi Baklouti ${ }^{1}$ \\ ${ }^{1}$ Centre de Génétique Moléculaire et Cellulaire, CNRS UMR 5534, Université Lyon 1; Bât. Gregor Mendel, 16, rue R. Dubois, 69622 \\ Villeurbanne, France
}

\begin{abstract}
Sustained expression of the Spi-1/PU.1 and Fli-1 oncoproteins blocks globin gene activation in mouse erythroleukemia cells; however, only Spi-1/PU.1 expression inhibits the inclusion of exon 16 in the mature 4.1R mRNA. This splicing event is crucial for a functional 4.1R protein and, therefore, for red blood cell membrane integrity. This report demonstrates that Spi-1/PU.1 downregulation induces the activation of TRIM10/hematopoietic RING finger 1 (HERF1), a member of the tripartite motif (TRIM)/RBCC protein family needed for globin gene transcription. Additionally, we demonstrate that TRIM10/HERF1 is required for the regulated splicing of exon 16 during late erythroid differentiation. Using inducible overexpression and silencing approaches, we found that: (1) TRIM10/HERF1 knockdown inhibits hemoglobin production and exon splicing and triggers cell apoptosis in dimethylsulfoxide (DMSO)-induced cells; (2) TRIM10/HERF1 upregulation is required but is insufficient on its own to activate exon retention; (3) Fli-1 has no effect on TRIM10/HERF1 expression, whereas either DMSO-induced downregulation or shRNA-knockdown of Spi-1/PU.1 expression is sufficient to activate TRIM10/HERF1 expression; and (4) Spi-1/PU.1 knockdown triggers both the transcription and the splicing events independently of the chemical induction. Altogether, these data indicate that primary Spi-1/PU.1 downregulation acts on late erythroid differentiation through at least two pathways, one of which requires TRIM10/HERF1 upregulation and parallels the Spi-1/PU.1-induced Fli-1 shutoff regulatory cascade.
\end{abstract}

Keywords: alternative splicing, erythroid differentiation, HERF1, oncogene, protein 4.1R, Spi-1/PU.1, TRIM10 Cell Research (2008) 18:834-845. doi: 10.1038/cr.2008.68; published online 17 June 2008

\section{Introduction}

Hematopoietic RING finger 1 (HERF1) was originally described as a downstream component of a regulatory cascade initiated by AML1/core binding factor $(\mathrm{CBF} \beta)[1]$, a transcription factor complex essential for the formation of the definitive hematopoietic system [2,3]. Additionally, HERF1 was independently identified by a comprehensive genomics approach as TRIM10, a member of the N-terminal RING finger/B-box/coiled coil (RBCC) or tripartite motif

Correspondence: Faouzi Baklouti

Tel: +33-4-72-43-29-28; Fax: +33-4-72-43-26-85

E-mail: faouzi.baklouti@univ-lyon1.fr

Abbreviations: dimethylsulfoxide (DMSO); hematopoietic RING finger 1 (HERF1); hexamethylene bisacetamide (HMBA); mouse erythroleukemia (MEL); N-terminal RING finger/B-box/coiled coil (RBCC); tripartite motif (TRIM)

Receiced 2 December 2007; revised 26 March 2008; accepted 11 April 2008; published online 17 June 2008
(TRIM) family of proteins ([4-6] and references therein). Therefore, we will refer to this protein as TRIM10/HERF1, as previously suggested [4]. TRIM/RBCC proteins are involved in a variety of processes, including apoptosis, cell cycle regulation, senescence, cell growth, viral response, cell differentiation, meiosis, and vesicular transport. Genes belonging to this family are implicated in several human diseases that vary from cancer to rare genetic syndromes [4]. The RING domains function as protein-protein interaction modules and mediate E3 ubiquitin ligase activity by promoting E2-dependent ubiquitin conjugation [5]. Such homo- or hetero-dimeric protein-protein interactions are needed for PML growth suppressor, apoptotic, and antiviral activities [7].

Induction of mouse erythroleukemia (MEL) cells to terminally differentiate using dimethylsulfoxide (DMSO) is associated with a 30-fold increase in TRIM10/HERF1 expression. Conversely, inhibition of TRIM10/HERF1 expression blocks hemoglobin production in MEL cells, even 
in the presence of DMSO, suggesting that TRIM10/HERF1 must play a key role in definitive erythroid development [1]. Insertion of the Friend viral genome upstream of the Spi-1/PU.1 locus in MEL cells leads to constitutive upregulation of the Spi-1/PU.1 gene, uncontrolled proliferation, and a subsequent block in the differentiation of the affected erythroblasts $[8,9]$. Spi-1/PU.1 is exclusively expressed in hematopoietic cells, and it is a potent regulator of many hematopoietic lineages. In fact, Spi-1/PU.1 expression is critical for the maturation of myeloid and B lymphoid lineage cells $[10,11]$, for mast and dendritic cell differentiation, and for the early stages of T lymphopoiesis [12-14]. Overexpression of Spi-1/PU.1 blocks erythroid differentiation [15-17]. However, Spi-1/PU.1 is expressed at low levels in early erythroid cells. Spi-1/PU.1 mRNA is detected in bipotent erythroid-megakaryocytic progenitors, and the protein is detected in primary murine erythroblasts [18]. Moreover, it has been shown that Spi-1/PU.1 expression maintains the self-renewal capacity of immature erythroid precursors $[19,20]$.

In addition to its role as an oncogenic transcription factor, Spi-1/PU.1 is also involved in pre-mRNA splicing regulation, where it interacts with splicing factors ([21, 22] and reviewed in [23]). In previous studies, we have shown that chemical induction of MEL cell differentiation can properly regulate the erythroid-specific splicing of the alternative exon 16 of the 4.1R mRNA. Exon 16 is preferentially excluded from the 4.1R mRNA in uninduced cells, but predominantly included in differentiating cells. Inclusion of the 21-amino-acid sequence motif encoded by exon 16 at the $\mathrm{N}$-terminus of the $10 \mathrm{kDa}$ spectrin-actinbinding (SAB) domain is required for a functional $4.1 \mathrm{R}$ in mature red cells ([24] and references therein). More recently, we have demonstrated that two members of the ETS family of transcription factors, the oncogenes Spi-1/PU.1 and Fli-1, block MEL cell erythroid differentiation as evidenced by hemoglobin synthesis, but only Spi-1/PU.1 inhibits the regulated splicing of exon 16, suggesting that downregulation of Spi-1/PU.1 triggers erythroid terminal differentiation through at least two different pathways, one of which is independent of the Spi-1-induced Fli-1 regulatory cascade [25].

To address the role of TRIM10/HERF 1 in terminal erythroid differentiation, we used both globin gene activation as a transcriptional event and exon 16 selection as an mRNA maturation event to determine if TRIM10/HERF1 is a primary target of chemical derepression during MEL cell differentiation. We found that TRIM10/HERF1 expression activates both hemoglobin production and the regulated splicing of exon 16. Conversely, TRIM10/HERF1 downregulation inhibits both events and triggers cell apoptosis in the presence of a chemical inducer, suggesting a critical role for TRIM10/HERF1 in terminal erythroid differentiation and survival. Finally, we showed that TRIM10/HERF1 expression is independent of the regulatory cascade involving Spi-1 activation of Fli-1; yet, TRIM10/HERF1 upregulation requires Spi-1/PU.1 downregulation in differentiating MEL cells, but TRIM10/HERF1 expression itself is not sufficient to properly regulate $4.1 \mathrm{R}$ splicing.

\section{Results}

TRIM10/HERF1 is upregulated in differentiating erythroid cell lines regardless of the type of differentiation inducer used

It has been shown that TRIM10/HERF1 expression increases dramatically in MEL cells following treatment with DMSO [1]. To determine if this enhanced expression is a direct effect of DMSO or the result of an erythroid differentiation program activated by a differentiation inducer, we measured the change in TRIM10/HERF1 steady-state mRNA expression levels in two different spleen-focus forming virus (SFFV)-MEL cell lines following treatment with either of the chemical inducers (DMSO or hexamethylene bisacetamide (HMBA)) or the physiological inducer erythropoietin (EPO) (Figure 1A). We found that TRIM10/HERF1 mRNA expression was increased in SFFV 745A cells after DMSO or HMBA treatment, but not following EPO treatment. In EPO-responsive SKT6 cells, however, TRIM10/HERF1 mRNA expression was upregulated with differentiation induction by DMSO, HMBA or EPO. Consistent with these results, western blot analysis using an anti-TRIM10/HERF1 antibody revealed that the protein level was also increased in chemically induced 745-A cells and in SKT6 cells treated with either chemical inducer or EPO (Figure 1B). These results suggest that TRIM10/HERF1 upregulation is associated with the cells' potential to undergo terminal erythroid differentiation and is not a direct result of the chemical induction.

TRIM10/HERF1 is necessary but insufficient to activate hemoglobin production and exon 16 erythroid-specific splicing

Knowing that TRIM10/HERF1 upregulation during terminal erythroid differentiation induces globin gene expression [1], we aimed to determine if TRIM10/HERF1 expression also modulates the erythroid splicing of exon 16. MEL cell clones stably transfected with a tetracycline (Tet)-inducible vector expressing sense TRIM10/HERF1 RNA (HERF $(+)$ cells) were tested for their ability to regulate exon 16 splicing. As shown in Figure 2, overexpression of TRIM10/HERF1 alone fails to activate exon inclusion in the absence of chemical induction, suggesting that TRIM10/HERF1 is not sufficient to induce exon 16 
A

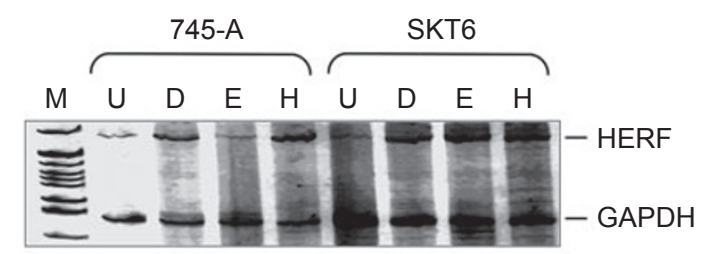

B

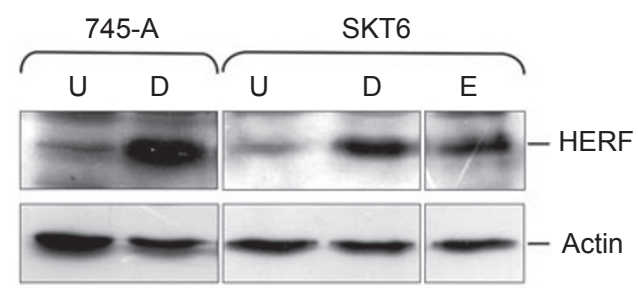

Figure 1 TRIM10/HERF1 expression during late erythroid differentiation. (A) TRIM10/HERF1 mRNA expression was assessed by RT-PCR using a limited number of cycles (28 cycles). RNA was obtained from EPO-unresponsive (745-A) or EPO-responsive (SKT6) cells. Cells were collected prior to induction (U, uninduced) or four days after induction to differentiation using DMSO (D), HMBA $(\mathrm{H})$, or EPO (E). GAPDH RNA was amplified in duplex to serve as an internal control. (B) Western blot analysis of TRIM10/ HERF1 expression in 745-A and SKT6 cells in the absence (U) or presence of DMSO (D) or EPO (E). Actin signal served as a loading control.

inclusion. Interestingly, these cells remained benzidinenegative, suggesting that they were incapable of producing hemoglobin tetramers. These findings are consistent with the previous reports stating that exon-16-regulated splicing occurs only in cells undergoing terminal erythroid differentiation, i.e., cells with the potential to produce hemoglobin [25]. Most remarkably, when TRIM10/HERF1 overexpression is combined with DMSO (or HMBA, data not shown) treatment, exon 16 inclusion is enhanced by almost twice as much as with DMSO alone (Figure 2B). These data are consistent with a cumulative effect of the chemically induced endogenous TRIM10/HERF1 expression and the induced overexpression of the transfected TRIM10/HERF1; therefore, TRIM10/HERF1 acts in a dose-dependent manner. Collectively, these experiments suggest that TRIM10/HERF1 activates the erythroid splicing event; however, it might act cooperatively with another factor (or factors), the expression of which also depends on the chemical induction.

\section{TRIM10/HERF1 knockdown triggers differentiating cell} death by apoptosis

To emphasize the importance of TRIM10/HERF1 in terminal erythroid differentiation, we analyzed the impact of Tet-off inducible antisense TRIM10/HERF1 mRNA in
HERF1(-) cells. Inhibition of TRIM10/HERF1 expression combined with DMSO (or HMBA) treatment led to a massive cell death after five days of treatment. Interestingly, cell viability was not affected during the first two days of induction, but starting from the third day the proportion of dead cells increased dramatically (Figure 3A) as cell proliferation rates slowed (Figure $3 \mathrm{~B}$ ). A methotrexate

A

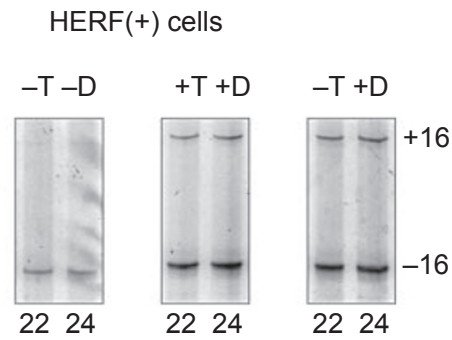

Cycles 2224

2224

2224

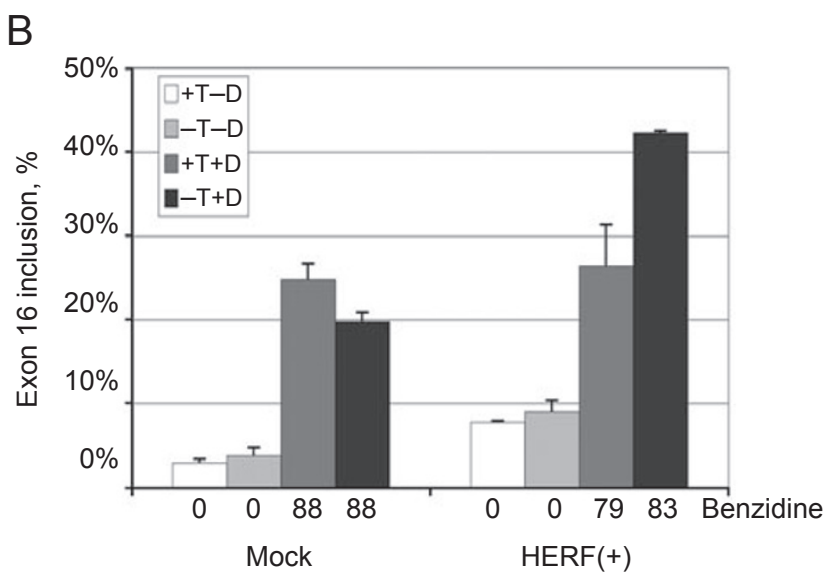

Figure 2 TRIM10/HERF1 overexpression activates erythroid terminal differentiation in DMSO-induced MEL cells. Hemoglobinproducing cells and exon 16 splicing were monitored in $\operatorname{HERF}(+)$ cells in the presence or absence of DMSO. (A) Acrylamide gel electrophoresis of semi-quantitative RT-PCR, performed around exon 16 with a limited number of cycles (22 and 24). M, size marker. +16 and -16 indicate the inclusion or the exclusion of exon 16 , respectively. $+T$ and $-T$ indicate the presence or absence of tetracycline. $+D$ and $-D$ refer to the presence or absence of the chemical inducer DMSO. (B) Quantitative analysis of exon 16 splicing before $(-D)$ and 5 days after DMSO (+D) treatment in MEL cells expressing $(-T)$ or not expressing TRIM10/HERF1 sense $(+T)$. Exon inclusion is expressed as a percentage of the following ratio: $(+16) /((+16)+(-16))$. A mean of hemoglobin-containing cells is indicated as percentage of benzidine-positive cells for each experimental condition. Similar results were obtained with HMBA treatment. Mock cells are cells transfected with the empty vector (see Materials and Methods). Two different HERF (+) clones were tested. Different sets of experiments were performed, and each correspond to a different DMSO-induction. One representative set of experiments is shown. Exon inclusion is indicated as mean $\pm S D$. 
A

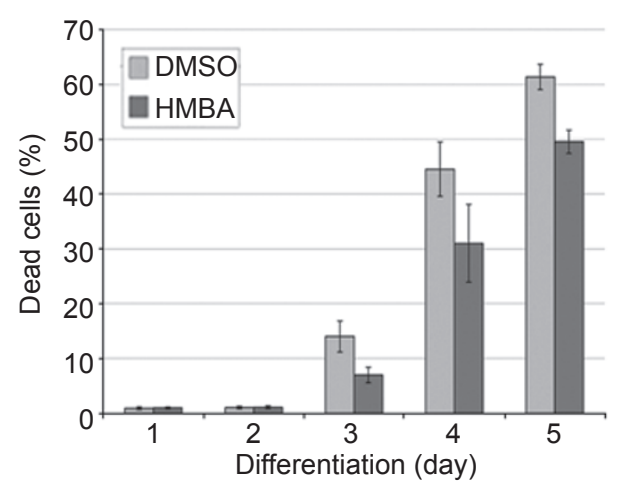

B

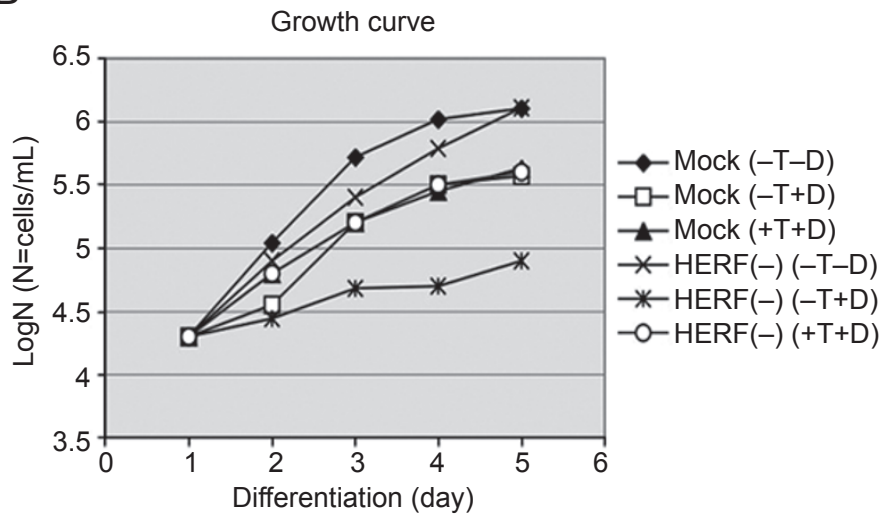

C

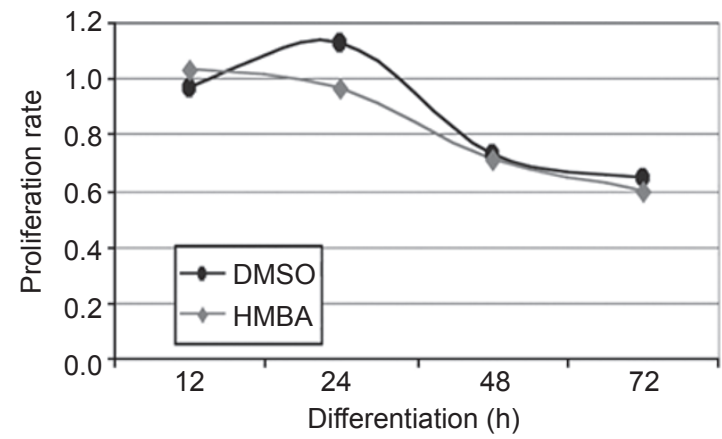

D

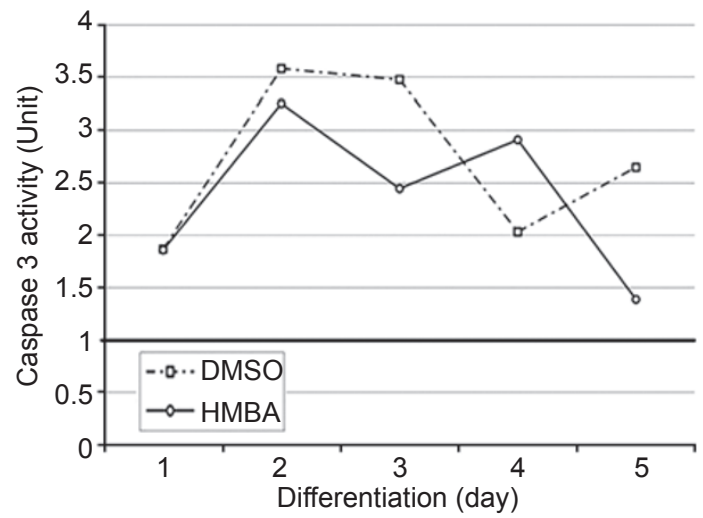

$E$

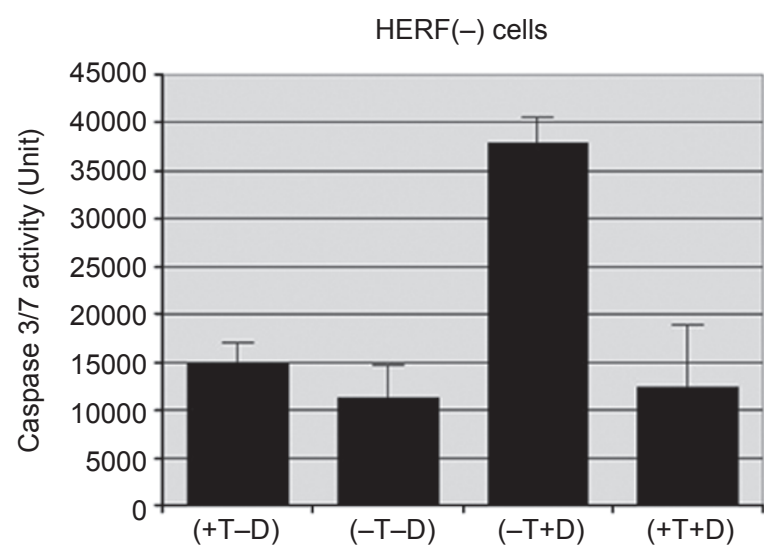

Figure 3 Impact of TRIM10/HERF1 downregulation on cell viability. (A) Time-course analysis of cell viability using trypan blue staining procedure. MEL cells expressing antisense TRIM10/HERF1 RNA were induced to terminally differentiate for five days using either DMSO or HMBA. Data correspond to mean \pm SD of 3 different experiments. (B) Growth curves for HERF(-) cells and mock cells cultured in the absence $(-)$ or presence $(+)$ of DMSO (D) and tetracycline (T). (C) MTT assay in HERF $(-)$ cells. The metabolic activity is expressed as the ratio of absorbance of HERF(-) cells over mock cells. Cellular metabolism of $\operatorname{HERF}(-)$ cells decreases after $48 \mathrm{~h}$ of DMSO exposure. The experiment was performed four times with similar results, and one representative experiment is shown. (D) Time-course analysis of caspase-3 activity during DMSO or HMBA treatment of $\operatorname{HERF}(-)$ cells. Caspase-3 activity is expressed as a ratio of $\mathrm{HERF}(-)$ cells over mock cells (constant curve with caspase-3 activity=1). Data were confirmed from 3 different experiments. (E) Caspase $3 / 7$ activity in untreated $(-) H E R F(-)$ cells and five days after exposure (+) to DMSO (D) and/or tetracycline $(T)$. The experiment was performed twice, and Caspase $3 / 7$ activity is presented as mean \pm SD. 
(MTT) assay was also performed on HERF1(-) cells before and after exposure to chemical induction, which led to the suggestion that TRIM10/HERF1 downregulation affects the growth of cells committed to late erythroid differentiation (Figure 3C). This observation suggests that TRIM10/HERF1 is required for the viability of cells undergoing terminal erythroid differentiation. To determine if cell death is apoptotic in nature, caspase- 3 activity was estimated during the five days of exposure to the chemical inducer (Figure 3D). In the absence of a chemical inducer, cells that express TRIM10/HERF1 antisense did not show a significant change in caspase-3 activity. However, when TRIM10/HERF1 expression was blocked in the induced cells, caspase- 3 activity nearly doubled from the first 24 hours. During the following days, it increased further in these cells to reach about 3.5 times the caspase- 3 activity in induced cells with unaffected TRIM10/HERF1 expression. One-step assays for caspase-3 and -7 , both involved

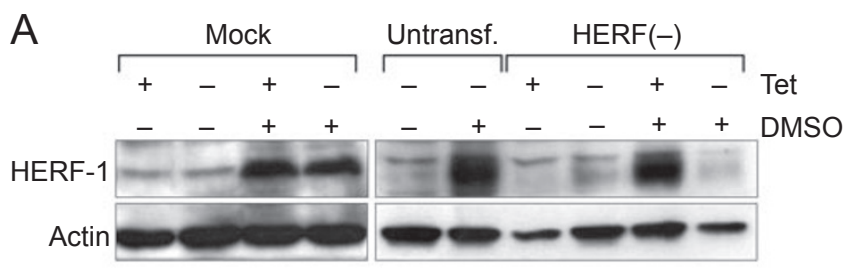

B

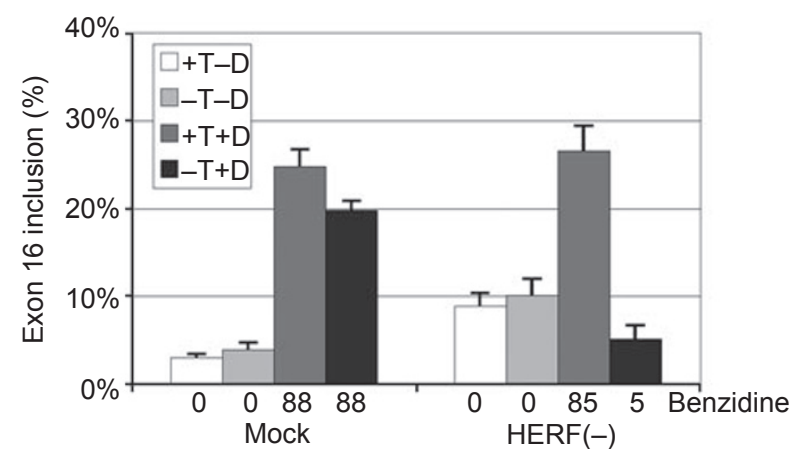

Figure 4 TRIM10/HERF1 knockdown inhibits specific events of late erythroid differentiation. (A) Western blot analysis using an anti-TRIM10/HERF1 antibody to assess TRIM10/HERF1 expression in HERF(-) cells as compared with untransfected cells or cells transfected with the empty vector (Mock). Actin immunoblotting served as a loading control. (B) Hemoglobin synthesis and exon 16 splicing efficiency were analyzed in HERF(-) cells and MEL cells transfected with the empty vector (mock). Quantitative analysis of exon 16 splicing before (-D) and five days after DMSO (+D) treatment of MEL cells expressing Herf1 antisense $(-T)$ or not $(+T)$. Two different HERF (-) clones were tested. Different sets of experiments were performed, and each correspond to a different DMSO-induction. One representative set of experiments is shown. Exon inclusion is indicated as mean $\pm S D$.

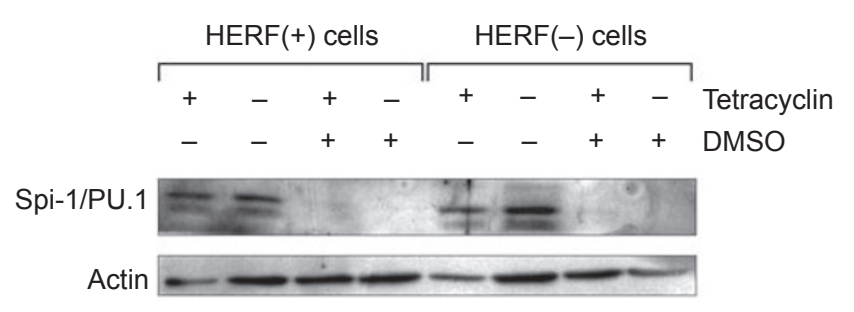

Figure 5 Effect of TRIM10/HERF1 on Spi-1/PU.1 expression. Spi-1/PU.1 was assessed by western blot analysis in $\mathrm{HERF}(+)$ and HERF(-) cells in the presence or absence of DMSO treatment and in the presence or absence of tetracycline. Actin was used as a protein loading control.

in the execution of apoptosis, further demonstrated an enhanced activity in $\operatorname{HERF}(-)$ cells once DMSO was added to induce cell differentiation and doxycycline was concomitantly withdrawn to inactivate TRIM10/HERF1 expression (Figure 3E). Collectively, these experiments suggest that knockdown of TRIM10/HERF1 in differentiating cells triggers cell death by apoptosis.

TRIM10/HERF1 knockdown blocks both hemoglobin production and exon 16 erythroid-specific splicing

In spite of the massive apoptosis following TRIM10/ HERF1 knockdown, the few remaining live cells were analyzed for their hemoglobin production and the regulated splicing of exon 16. TRIM10/HERF1 protein expression was first assessed by immunoblotting to ascertain the specific effect of antisense TRIM10/HERF1 RNA following Tet-off induction (Figure 4A). In DMSO-uninduced cells, TRIM10/HERF1 expression is low, so Tet-off induction of antisense TRIM10/HERF1 RNA had no effect on exon inclusion in HERF $(-)$ cells compared to mock cells (Figure 4B). However, upon DMSO exposure, we reproducibly observed that TRIM10/HERF1 downregulation blocks both hemoglobin production and exon 16 inclusion (Figure 4B). These results emphasize the crucial role of TRIM10/HERF1 in terminal erythroid differentiation, and that hemoglobin synthesis and the regulated splicing of exon 16 are induced via a pathway that requires TRIM10/HERF1 upregulation.

This potential role in splicing regulation prompted us to test the impact of TRIM10/HERF1 downregulation on specific splicing factors, such as hnRNP A1, which is the only factor known to inhibit exon 16 inclusion [26]. Immunoblotting experiments showed that the modulation of TRIM10/HERF1 expression does not seem to affect hnRNP A1 expression (Supplementary information, Figure S1), suggesting that TRIM10/HERF1 most likely acts through an hnRNP A1-independent pathway. Given that TRIM10/ HERF1 is exclusively expressed in the cytoplasm [5], it seems unlikely that it would act on splicing through a direct 
A
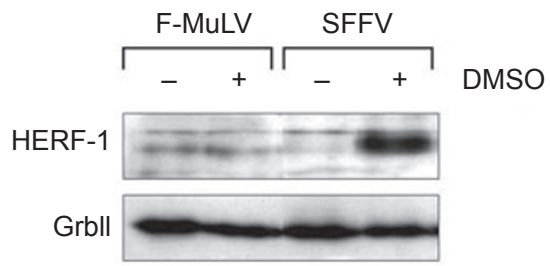

B

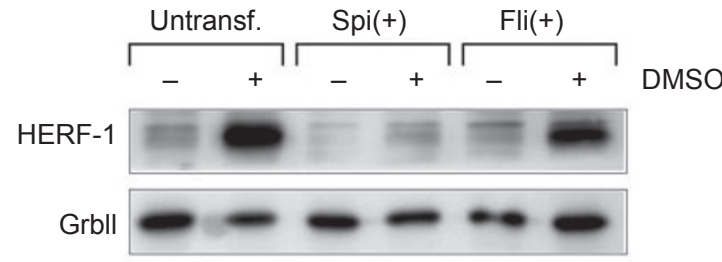

C

Figure 6 TRIM10/HERF1 upregulation requires Spi-1/PU.1 but not Fli-1 downregulation. (A) Western blot analysis of TRIM10/HERF1 expression in F-MuLV and SFFV cells in the presence or absence of DMSO treatment. TRIM10/HERF1 is upregulated only in cells with the potential to terminally differentiate in response to DMSO induction. (B) TRIM10/HERF1 expression analysis by western blot in SFFV cells overexpressing either Spi-1/PU.1 (Spi(+)) or Fli-1 $(\mathrm{Fli}(+))$. The data are compared with TRIM10/HERF1 expression in untransfected SFFV cells (untransf.). Immunoblotting with a Grbll antibody serves as a control to normalize TRIM10/HERF1 expression levels. (C) TRIM10/HERF1 mRNA analysis using RT$\mathrm{PCR}$ in $\mathrm{Spi}(+)$ cells before and after DMSO exposure. Actin was used as an internal control. 745-A cells were used as control cells (Ctr). HERF1/Actin ratio is expressed as mean \pm SD, collected from 3 different experiments.

interaction with the pre-mRNA molecule.

TRIM10/HERF1 does not affect Spi-1/PU.1 expression in MEL cells

Most observations have been collected from experiments using MEL cell clones derived from mice infected with a replication-defective variant of the Friend virus complex, SFFV. In these cells, Spi-1/PU.1 and Fli-1 oncogenes are constitutively overexpressed and treatment with a chemical inducer, such as DMSO, results in a rapid shutoff of both oncogenes and an induction of erythroid terminal differentiation. However, while Spi-1/PU.1 and Fli-1 both inhibit hemoglobin production in DMSO-treated cells, only Spi-1/PU.1 inhibits exon 16 splicing regulation [25]. Therefore, we addressed the possible link between Spi1/PU.1 downregulation and TRIM10/HERF1 activation during late erythroid development. Spi-1/PU.1 was assessed by western blot analysis in $\operatorname{HERF}(+)$ and $\operatorname{HERF}(-)$ cells (Figure 5). Forced expression of TRIM10/HERF1 in HERF $(+)$ cells did not affect Spi-1/PU.1 protein levels, clearly indicating that Spi-1/PU.1 expression remains unchanged in the absence of chemical induction and decreases upon exposure to DMSO, independently of the Tet-off induction of TRIM10/HERF1 overexpression. Similarly, Tet-off blockade of TRIM10/HERF1 expression in HERF(-) cells did not affect Spi-1/PU.1 protein levels, the downregulation of which was again strictly triggered by chemical induction (Figure 5). Even though Spi-1/PU.1 downregulation occurred in chemically induced HERF1(-) cells, these cells failed to produce hemoglobin and were unable to regulate exon 16 inclusion (Figure 4). Collectively, these results suggest that TRIM10/HERF1 has no effect on Spi-1/PU.1 expression in erythroleukemia cells, but its presence is required for specific regulatory events of terminal erythroid differentiation.

TRIM10/HERF1 upregulation strictly correlates with Spi1/PU.1 downregulation

To determine if TRIM10/HERF1 upregulation occurs downstream of the Spi-1/PU.1-induced Fli-1 cascade [27] or through activation of an alternative pathway that still requires Spi-1/PU.1 inactivation, we examined TRIM10/ HERF1 expression in F-MuLV cells, which overexpress Fli-1 but not Spi-1/PU.1. In these cells, the TRIM10/ HERF1 protein level was low and similar to that observed in uninduced SFFV cells (Figure 6A). This pattern of expression remains unchanged following DMSO exposure, further indicating that TRIM10/HERF1 activation is not a direct effect of the chemical treatment.

We next tested the impact of enforced expression of Spi-1/PU.1 or Fli-1 on TRIM10/HERF1 expression in SFFV cells (Figure 6B). Forced expression of Spi-1/PU.1 (Spi $(+)$ cells) blocks MEL erythroid differentiation as well as exon 16 regulated splicing following DMSO cell induction [25]. Immunoblotting for TRIM10/HERF1 displayed a minimal increase, if any, in protein expression in DMSOtreated Spi $(+)$ cells compared to untreated cells, suggesting that TRIM10/HERF1 full activation requires Spi-1/PU.1 
A

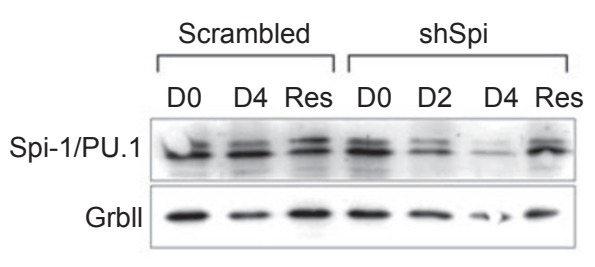

B

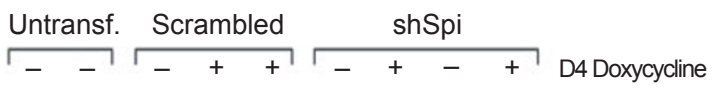

$-+\ldots++\ldots++$ DMSO

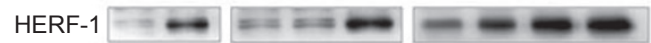

$\begin{aligned} \mathrm{Spi}-1 / \mathrm{PU} 1 & =-1=- \\ \mathrm{Grbll} & --\infty-\infty\end{aligned}$

C

HERF1 mRNA steady state

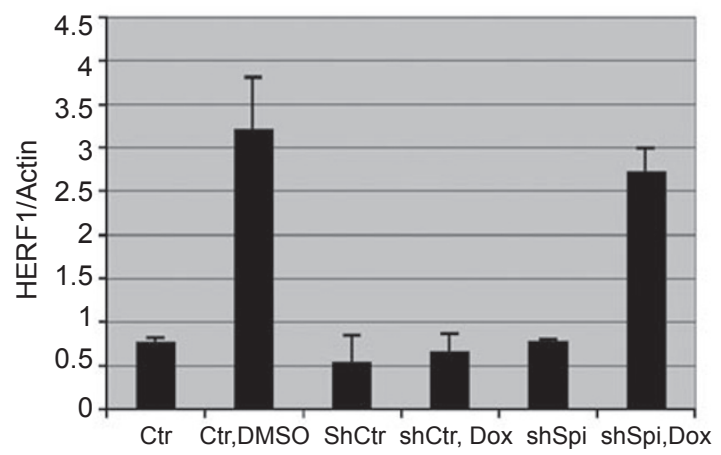

Figure 7 Spi-1/PU.1 knockdown triggers TRIM10/HERF1 upregulation. (A) shRNA-mediated knockdown of Spi-1/PU.1. Spi-1/PU.1 expression is monitored before (D0), two (D2) and four (D4) days after doxycycline treatment to induce shRNA expression. Spi-1/PU.1 expression was also analyzed four days after doxycycline removal from the medium (rescue, Res.). Cells were transfected with either a specific Spi-1/PU.1 shRNA or a scrambled shRNA sequence. (B) Effect of Spi-1/PU.1 knockdown on TRIM10/HERF1 expression. TRIM10/HERF1 expression was analyzed by western blot analysis in untransfected cells (untransf.) or cells transfected with either a scrambled sequence (scrambled) or a Spi-1/PU.1 shRNA specific sequence (shSpi). Protein expression was examined after the cells received four days of doxycycline treatment in combination with or without DMSO chemical induction. (C) TRIM10/HERF1 mRNA analysis using RT-PCR. Samples correspond to untransfected cells (Ctr), mock cells transfected with scrambled shRNA vector (shCtr) or cells expressing doxycycline-inducible shSpi (ShSpi). Note that TRIM10/HERF1 mRNA increases only when Spi-1/PU.1 is downregulated either via DMSO induction or shRNA knockdown. HERF1/Actin mRNA ratio is expressed as mean \pm SD, collected from 3 different experiments. inactivation. Interestingly, constitutive overexpression of Fli-1 in induced SFFV cells, which was shown to affect hemoglobin synthesis but not exon 16 splicing [25], does not seem to affect TRIM10/HERF1 expression (Fli-1+ cells, Figure 6B), offering additional evidence suggesting that TRIM10/HERF1 upregulation is strictly governed by Spi-1/PU.1 inactivation induced by DMSO. In agreement with protein expression, TRIM10/HERF1 steady-state mRNA increased in strict correlation with Spi-1/PU.1 downregulation, and, as shown in Figure $6 \mathrm{C}$, constitutive overexpression of Spi-1/PU.1 results in TRIM10/HERF1 repression following DMSO cell treatment. Altogether, these observations suggest that TRIM10/HERF1 expression is not correlated with Fli-1, but it is negatively regulated by Spi-1/PU.1 expression in MEL cells.

\section{TRIM10/HERF1 is activated in Spi-1/PU.1 knockdown cells}

We next determined if TRIM10/HERF1 upregulation is the result of Spi-1/PU.1 inactivation or, instead, if it is dependent on a different pathway triggered by the inducer. To address this issue, endogenous Spi-1/PU.1 was silenced using an inducible shRNA system (see Materials and Methods, and [28]). Endogenous Spi-1/PU.1 expression was assessed to ascertain the specificity of the shRNA and to determine if the effect of the transfected shRNA was reversible (Figure 7A). Immunoblots revealed an increase in TRIM10/HERF1 expression associated with DMSOinduced Spi-1/PU.1 downregulation in untransfected cells and in cells transfected with either the scrambled shRNA or Spi-1/PU.1 shRNA (Figure 7B). Most importantly, TRIM10/HERF1 expression, at both the protein (Figure 7B) and mRNA (Figure 7C) levels, increased in the absence of DMSO concomitantly with shRNA-mediated Spi-1/PU.1 knockdown. These results strongly suggest that TRIM10/ HERF1 upregulation is a result of Spi-1/PU.1 downregula-

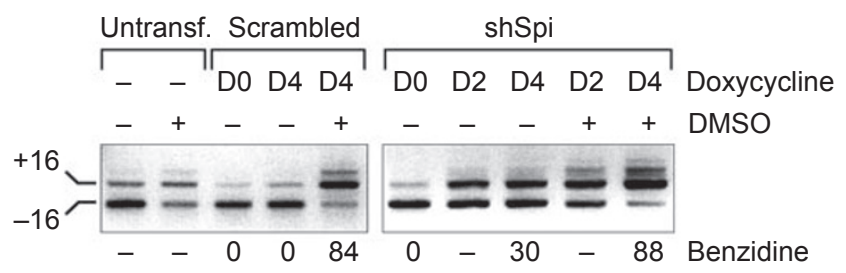

Figure 8 Spi-1/PU. 1 knockdown triggers hemoglobin production and exon 16 erythroid-specific splicing. RT-PCR analysis of 4.1R mRNA was performed in untransfected MEL cells (untransf.) and in cells transfected with either scrambled (scrambled) or Spi-1/ PU.1shRNA-specific sequence (shSpi). Cells were collected before (D0), two (D2) or four (D4) days after doxycycline treatment in combination with or without DMSO induction. Hemoglobin-containing cells are indicated as a percentage of benzidine-positive cells. 
tion, and they further support the idea that TRIM10/HERF1 activation is not a direct effect of DMSO treatment.

The Spi-1/PU.1-induced TRIM10/HERF1 cascade activates both hemoglobin production and exon 16 erythroidspecific splicing

We have established that both DMSO-induced Spi-1/ PU.1 downregulation [25] and TRIM10/HERF1 activation (this report) are required to initiate exon 16 erythroid splicing regulation. These results led us to ask if the cascade involving Spi-1/PU.1 shutoff-mediated activation of TRIM10/HERF1 is able to activate, independently of DMSO induction, the two specific events of terminal erythroid differentiation, hemoglobin production, and exon 16-regulated splicing. Spi-1/PU.1 silencing was often accompanied by a spontaneous differentiation of around $20-30 \%$ of MEL cells, as evidenced by the benzidinepositive cells found within different sets of experiments (Figure 8). These findings suggest a significant production of hemoglobin in these cells, in the absence of DMSO treatment, and, therefore, resulting only from the decrease in Spi-1/PU.1 level. Such "spontaneous differentiation" has been recently reported using a different Spi-1/PU.1 silencing approach [29].

Exon 16 inclusion was also monitored in shSpi cells during induction of Spi-1/PU.1 shRNA expression in the presence of doxycycline. Figure 8 shows an increasing amount of mature 4.1R mRNA containing exon 16, suggesting an activation of the erythroid regulation of exon splicing. This increase coincides with a sequential decline over time of Spi-1/PU.1 and a concomitant increase of TRIM10/HERF1 expression (Figure 7B). These findings suggest that exon 16 inclusion efficiency correlates with the silencing capacity of the induced shRNA. The effect of Spi-1/PU.1 inhibition therefore acts in a dose-dependent manner both on TRIM10/HERF1 expression and on erythroid 4.1R mRNA splicing. Together, these experiments suggest that the cascade involving Spi-1/PU.1 downregulation-mediated activation of TRIM10/HERF1 triggers terminal erythroid differentiation, independently of DMSO induction.

\section{Discussion}

The later stages of red cell development are accompanied by major changes in gene expression regulation that are best characterized by hemoglobin synthesis, all of which contribute to optimize the specific functions of this highly specialized cell. Among these functions, one of the most important is the establishment of a particular protein architecture, the red cell skeleton, that allows the cell membrane a high capacity of flexibility and deformability [30]. Protein 4.1R is a key component of the red cell skeleton, and it is only fully functional in mature red cells because of the inclusion of a 21-amino-acid sequence that is regulated by an alternative splicing event occurring at a late stage of erythroid development [24, 31].

\section{Erythroid terminal differentiation is activated by TRIM10/} HERF1 through a Spi-1/PU.1 shutdown-dependent pathway

Continuous Spi-1/PU.1 expression is not compatible with erythroid differentiation. In fact, Spi-1/PU.1 protein is present at a low level in normal proerythroblasts, and its production is shut off as the cells mature [18]. However, in Friend-infected erythroleukemic cells, Spi-1/PU.1 protein is expressed at a high level [16]. It is thought that persistent and elevated expression of Spi-1/PU.1 may be required to maintain the leukemic phenotype. The rapid decline in Spi1/PU.1 expression following chemical induction appears to be an early and important event required for terminal erythroid differentiation of MEL cells [16]. Using an inducible shRNA strategy, Spi-1/PU.1 knockdown concomitantly results in cell growth arrest, hemoglobin production and activation of exon 16 erythroid-specific splicing.

As other oncogenic transcription factors, Spi-1/PU.1 is involved in pre-mRNA splicing regulation [23]. We have demonstrated that splicing of exon 16 in differentiating MEL cells is regulated within an erythroid terminal differentiation program and that constitutive overexpression of either Spi-1/PU.1 or Fli-1 in SFFV cells inhibits hemoglobin synthesis, but only that of Spi-1/PU.1 affects the regulated splicing [25]. These data were consistently supported by the Spi-1/PU.1 knockdown experiments. These findings suggest that Spi-1/PU.1 oncogene downregulation induces late erythroid differentiation through at least two different pathways. The first pathway triggers Fli-1 downregulation and is needed for hemoglobin expression, but not for the

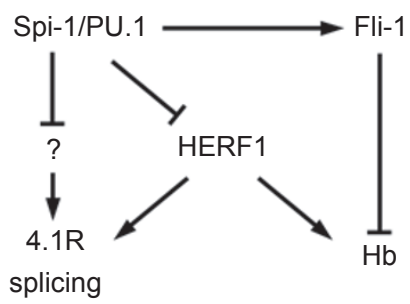

Figure 9 Schematic representation of regulatory pathways. TRIM10/HERF1 is needed for both hemoglobin production and for regulated exon 16 splicing during late erythroid development. Spi1/PU.1 triggers terminal differentiation arrest of MEL cells through at least two pathways involving either Fli-1 or TRIM10/HERF1. Overexpression of Spi-1/PU.1 blocks TRIM10/HERF1 expression but activates Fli-1 expression. Spi-1/PU.1 also acts on splicing through at least two mechanisms, one of which is TRIM10/HERF1dependent. Fli-1 has no effect on TRIM10/HERF1 expression or on regulated exon 16 splicing. 
regulated splicing event [25]. The second pathway parallels or overlaps the regulatory cascade involving Spi-1/PU.1 activation of Fli-1 and is required for both hemoglobin expression and erythroid splicing regulation. In the present work, we show that this second pathway is a cascade that includes TRIM10/HERF1 downstream of Spi-1/PU.1. We further show that Fli-1 does not affect TRIM10/HERF1 expression, yet upregulation of TRIM10/HERF1 requires the shutoff of Spi-1/PU.1 expression. Figure 9 is a schematic of a model of the regulatory pathways documented in this work and elsewhere.

Our data suggest that exon 16 erythroid-specific splicing requires TRIM10/HERF1 upregulation. However, when combined with Spi-1/PU.1 overexpression, TRIM10/HERF1 upregulation is incapable of inducing exon inclusion, again suggesting that Spi-1/PU.1 acts on exon 16 splicing through at least two different regulatory pathways, one of which is a TRIM10/HERF1-dependent pathway and the other is a TRIM10/HERF1-independent pathway that is still activated upon the shutoff of Spi1/PU.1 expression (Figure 9). Investigating the upstream effectors and the downstream targets of TRIM10/HERF1 expression will shed more light on these erythroid-specific regulation pathways.

TRIM10/HERF1 is critical for erythroid cell differentiation and its downregulation leads to cell death

Little is known about TRIM10/HERF1. Harada and colleagues [1] have shown that TRIM10/HERF1 increase is required for terminal differentiation of erythroid cells based on their ability to produce hemoglobin. Here, we demonstrated that this factor is also crucial for another late erythroid-specific event, the regulated splicing of exon 16 on $4.1 \mathrm{R}$ pre-mRNA. Furthermore, TRIM10/HERF1 knockdown in differentiating cells induces a marked growth arrest and apoptosis, but TRIM10/HERF1 knockdown by itself does not seem to affect proliferative MEL cells. However, the combination of TRIM10/HERF1 inhibition and DMSO-induction of cell differentiation leads to an arrest of cell proliferation and increased cell death by apoptosis. Additionally, HERF1(-) cells are unable to differentiate because of TRIM10/HERF1 knockdown. It is unlikely, however, that the cell death results from decreased Spi-1/PU.1 and/or Fli-1 expression since Spi-1/PU.1 downregulation alone does not stop cell proliferation of F-MuLV cells. This hypothesis is further supported by the concomitant early caspase-3 increase and Spi-1/PU.1 decrease in DMSOtreated HERF1(-) cells.

TRIM10/HERF1 is predominantly expressed during definitive erythropoiesis [1] and in embryonic liver [5]. However, TRIM10/HERF1 is also minimally expressed in adult liver, kidney, and colon [5]. Another member of the TRIM family, called Bloodthirsty (Bty), is required for erythropoiesis in the zebrafish [32]. However, bty knockdown reduced the abundance of several late-erythropoietic mRNAs, whereas expression of genes that mark early erythropoiesis, myelopoiesis and vasculogenesis, including Spi-1/PU.1 transcripts, was unaffected, suggesting that bty is required for terminal differentiation of erythroid progenitors of the primitive lineage [32]. The zebrafish bty gene seems to be orthologous to human RNF23/TRIM39 and not to TRIM10/HERF1. This is not surprising since the bty gene is most strongly expressed in the proerythroblast and normoblast [32], whereas TRIM10/HERF1 appears to be transcribed later in terminal differentiation [1]. However, both TRIM10/HERF1 and bty/RNF23/TRIM39 are members of the C-IV subfamily (B30.2-like or SPRY-containing domains) [6].

It has been proposed that $b t y /$ RNF23/TRIM39 and TRIM10/HERF1 contribute to a stage-specific cascade that targets transcriptional repressors involved in erythropoiesis for proteasome-based destruction via their putative ubiquitin ligase activity, subsequently releasing the proerythroblasts for erythroid maturation [32]. Regarding the mechanism by which TRIM10/HERF1 activates the erythroid-specific splicing of exon 16 , we believe that TRIM10/HERF1 acts indirectly within a cascade initiated by Spi-1/PU.1 downregulation, rather than directly on exon inclusion in the mature 4.1R mRNA. This hypothesis is supported by the following observations: (1) TRIM10/ HERF1 is expressed in cytoplasmic aggregates [5]; (2) TRIM10/HERF1 is not targeted to speckles, where some splicing factors are stored [5]; (3) TRIM10/HERF1 does not co-localize with spliceosomes (SC35) [5]; and (4) TRIM10/HERF1 does not contain RRM or RS domains; therefore, it is unlikely that TRIM10/HERF1 contribution to splicing decision acts through RNA binding.

In total, this work identifies TRIM10/HERF1 as a downstream target of the induced Spi-1/PU.1 oncogene downregulation in committed erythroid cells. Upregulation of TRIM10/HERF1 is required for a major splicing event, the achievement of which is critical for functional protein $4.1 \mathrm{R}$ and, therefore, for red cell membrane integrity. Moreover, these data indicate that the primary reduction of Spi-1/PU.1 expression acts on erythroid differentiation and exon erythroid-specific splicing through at least two pathways, one of which requires the upregulation of TRIM10/HERF1.

\section{Materials and Methods}

\section{Plasmid constructs and stably transfected cell lines}

MEL cell clones stably transfected with Tet-inducible sense $(\operatorname{HERF}(+)$ cells) or antisense $(\operatorname{HERF}(-)$ cells) TRIM10/HERF1 mRNA were kindly provided by Dr J Downing, and they have been 
prepared and used previously [1]. Briefly, MEL cells were first transfected with a pTET.TAK.HYG vector containing a Tet-inducible tet repressor DNA binding domain fused to VP16 activation domain and a constitutively expressed hygromycin selection gene. A selected clone was subsequently transfected with an Ahd-I-linearized pTET. HERF1.Neo or pTET.aHERF1.Neo vector, and clones expressing inducible sense or antisense TRIM10/HERF1 mRNA, respectively, were selected in the presence of G418, hygromycin, and Tet. In this Tet-off system, the Tet-controlled transactivator (TetR-VP16 fusion) binds the Tet-response element (TRE) and activates transcription in the absence of Tet. Conversely, addition of Tet prevents TetR-VP16 transactivator from binding to the TRE; therefore, induced expression of high levels of sense or antisense TRIM10/HERF1 mRNA is obtained only when Tet is removed from the culture medium. Mock cell clones contained the pTET.TAK.NEO vector. Two stably transfected clones have been tested for each construct.

Spi-1/PU.1 and Fli-1 overexpression was obtained as previously described from MEL cell clones stably transfected with plasmid constructs pHOOK-Spi-1/PU.1 and pEF-LAC-CAT-Fli-1, respectively [25].

\section{Inducible RNA interference}

To knockdown Spi-1/PU.1 expression, we used a doxycyclineinduced RNA polymerase III H1 promoter to drive Spi-1/PU.1 shRNA expression [28]. Cells expressing the Tet repressor (TetR) were transfected with the doxycycline-inducible vector. The addition of doxycycline to the culture medium induced expression of Spi-1/PU.1 shRNA, which triggers rapid downregulation of Spi1/PU.1 mRNA and protein. The original method [28] was modified as follows: MEL 745A cells were first stably transfected with pCDNA6/TR-EF1 $\alpha$ (kindly provided by Drs F Moreau-Gachelin and C Guillouf), conferring a constitutive expression of TetR. This vector was derived from pCDNA6/TR Invitrogen, where the CMV promoter was removed between the SpeI and NheI restriction sites and replaced with a eukaryotic promoter $(\mathrm{EF} 1 \alpha)$. The pTER vector contains a Zeocin-resistant gene and a Tet operator (TO) sequence located immediately downstream of the TATA box. In the absence of doxycycline, TetR binds to the TO sequence and represses shRNA transcription. Adding doxycycline to the culture medium results in the dissociation of the TetR-TO complex, which leads to the transcriptional activation of the shRNA by polymerase III. Cell clones were selected for TetR high expression using western blot screening, and the selected clones were transfected with a recombinant pTER vector [28], containing pre-annealed shSpi-1/PU.1 oligonucleotides inserted into the BglII/HindIII sites. Stably transfected clones were then selected using Zeocin. shSpi-1/PU.1 oligonucleotides were designed using Dharmacon software. We tested two Spi-1/PU.1 specific shRNAs: (5' ...GGA TGT TAC AGG CGT GCAA...TTG CAC GCC TGT AAC ATC C...3'; 5'...TCA TTA ACC ACT AAA GAC A...TGT CTT TAG TGG TTA ATGA....3') and a scrambled shRNA was used as a negative control ( $5^{\prime}$...CCC AAG TCT TAT CGT TGG T...ACC AAC GAT AAG ACT TGG G...3'). In all, $3 \mu \mathrm{g}$ of each of the complementary primers was annealed in $100 \mathrm{mM}$ potassium acetate, $30 \mathrm{mM}$ HEPES-KOH, $\mathrm{pH}$ 7.4, and $3 \mathrm{mM}$ magnesium acetate. Once annealed, the double-strand oligos were directly ligated into the HindIII/BglII sites of the pTER plasmid [28]. The resulting pTER recombinant plasmids were transformed and amplified in the DH5 $\alpha$ bacteria strain, and the inserts were subsequently sequenced to ascertain the integrity of the shSpi-1/PU.1 sequences.

\section{Cell culture, induction and transfection}

Two SFFV MEL cell lines were used: the EPO-nonresponsive subclone 745A and the EPO-responsive subclone SKT6 [25]. Moreover, two F-MuLV cell lines were used: NN10 and IW32 subclones, which are unable to undergo terminal differentiation upon chemical induction [25].

Cells were cultured in suspension in DMEM supplemented with $13 \%$ FBS. TRIM10/HERF1 transfected cells were cultured in suspension in $13 \% \mathrm{FBS} / \mathrm{DMEM}$ with $0.8 \mathrm{mg} / \mathrm{ml} \mathrm{G} 418,1 \mathrm{mg} / \mathrm{ml}$ hygromycin and $0.5 \mu \mathrm{g} / \mathrm{ml}$ Tet. Cells were induced to erythroid differentiation in the same media for five days in the presence of $1.8 \%$ DMSO, $5 \mathrm{mM}$ HMBA, or $2 \mathrm{U} / \mathrm{ml}$ EPO for MEL cells.

For Spi-1/PU.1 silencing experiments, MEL cells were stably transfected with a pCDNA6/TR-EF1 $\alpha$ vector conferring a constitutive expression of the TetR protein and pTER vectors containing shRNA sequences. Briefly, $5 \times 10^{5}$ cells were transfected with $2 \mu \mathrm{g}$ of plasmid DNA using Escort transfection reagent (Sigma Aldrich) in 6-well plates, according to the manufacturer's procedure. Cells were then seeded in 96-well plates with complete DMEM medium containing $600 \mu \mathrm{g} / \mathrm{ml}$ Zeocin and $40 \mu \mathrm{g} / \mathrm{ml}$ blasticidin to select stably transfected clones. We used $2 \mathrm{mg} / \mathrm{ml}$ doxycycline to induce the transcription of the shRNAs. Cells were collected for western blot analyses and RT-PCR analyses after two and four days of induction with doxycycline.

\section{Semi-quantitative RT-PCR}

Total RNA was extracted from cell lysates using the Trizol reagent (Invitrogen) according to the manufacturer's instructions. To evaluate exon 16 inclusion, semi-quantitative RT-PCR experiments were performed as detailed previously [33].

TRIM10/HERF1 RT-PCR was assessed using the following primers: herf forward: 5'-GCT GGC TTG GGG CTT TGT TTC A-3', and herf reverse: 5'-CAC TCG AGG GTC TGC TCC CAA GGG CGC TTG-3'. GAPDH and $\beta$-actin mRNAs were also amplified to serve as internal controls, using the following primers: gapdh forward: 5'-CAA CGA CCC CTT CAT TGA CCT C-3', gapdh reverse: 5'-TCT CGC TCC TGG AAG ATG GTG AT-3', $\beta$-actin forward: 5 '-GAG GCC CAG AGC AAG AGA GGT ATC C-3' and $\beta$-actin reverse: 5'-GGC ATA CAG GGA CAG CAC AGC CTG G-3'.

\section{Western blot}

Cultured cells were pelleted and lysed in RIPA buffer $(50 \mathrm{mM}$ Tris-HCl, $\mathrm{pH}$ 7.4, $150 \mathrm{mM} \mathrm{NaCl}, 20 \mathrm{mM}$ EDTA, 1\% triton X-100, $1 \%$ sodium deoxycholate and $0.1 \%$ SDS) supplemented with protease inhibitor cocktail "Complete Mini, EDTA free" (Roche, Meylan, France) and with 1/100 diluted phosphatase inhibitor ("Phosphatase Inhibitor cocktail II", Sigma), as recommended by the suppliers. In all, $15 \mu \mathrm{g}$ of proteins were resolved using either $9 \%$ or $12 \%$ SDSPAGE and blotted onto nitrocellulose membranes.

Spi-1/PU.1 content was estimated with an anti-Spi-1/PU.1 antibody (1:1 000) [34] and a goat anti-rabbit IgG antibody as a secondary antibody $(1: 10000)$. The immunoreactive proteins were revealed on autoradiography film using an ECL+ enhanced chemiluminescence detection kit (Amersham Biosciences AB, Uppsala, Sweden) according to the manufacturer's instructions. The antiTRIM10/HERF1 (generously provided by Dr J Downing) is a rabbit polyclonal antibody directed against the N-terminal 230 amino acids of TRIM10/HERF1 [1]; this antiserum was used at a 1/1 000 dilu- 
tion. hnRNP A1 expression was assessed using a specific antibody (Y-15; 1/1 000, Santa Cruz Biotechnology, Heidelberg, Germany) and a donkey anti-goat secondary antibody (1/10 000; Santa Cruz Biotechnology). A monoclonal antibody directed against mouse actin (Chemicon International, Temecula, CA, USA) or Grb2 protein (1/10 000 dilution of mouse monoclonal antibody, BD Transduction Laboratories) was used to standardize Spi-1/PU.1, hnRNP A1 and TRIM10/HERF1 signals.

\section{MTT colorimetric assay}

Cells were seeded in a 96-well plate at density of $5 \times 10^{4}$ cells per well. In all, $20 \mu \mathrm{l}$ of $5 \mathrm{mg} / \mathrm{ml}$ MTT dissolved in PBS was added to the cells and plates were incubated for $4 \mathrm{~h}$ at $37^{\circ} \mathrm{C}$. After the addition of the stop solution $(0.04 \mathrm{~N} \mathrm{HCl}$ in isopropanol), the absorbance at $580 \mathrm{~nm}(\mathrm{~A} 580 \mathrm{~nm})$ was measured on a microplate reader (Dynatech MR 5000, Dynatech Laboratories, Chantilly, VA, USA). The MTT test assesses cell metabolism based on the ability of the mitochondrial succinate-tetrazolium reductase system to convert the yellow compound MTT to a blue formazan dye. The optical density is proportional to the number of live metabolically active cells. The value of cellular activity was calculated as the ratio of A580 nm obtained for cells incubated without Tet over that obtained for cells cultured in the presence of Tet.

\section{Cell death analysis and caspase activity measurement}

Cell death was analyzed by the trypan blue staining procedure. Caspase-3 and -7 activities served to measure the cell apoptosis. Briefly, during five days of chemical induction of differentiation and/ or induction of TRIM10/HERF1 expression, about $5 \times 10^{5}$ cells were washed and resuspended in DMEM. Caspase- 3 activity alone was measured using the AcDEVD-AFC substrate (MBL, Japan) according to the manufacturer's instructions. The cell extracts were incubated at $37^{\circ} \mathrm{C}$ for $60 \mathrm{~min}$ with labeled substrate in reaction buffer. The absorbance of cleaved substrates was measured by spectrophotometry at $405 \mathrm{~nm}$ wavelength. Apoptosis activation is presented as the caspase activity ratio between tested cells and control cells, transfected with the empty vector. Caspase-3 and -7 activities were assessed using the Caspase-Glo 3/7 assay kit (Promega, Charbonnières, France). Luminescence measurements were performed using Victor 1420 Multilabel Counter (Perkin-Elmer, Coutaboeuf, France).

\section{Acknowledgments}

We gratefully thank Dr J Downing (Pathology Department, Saint Jude Children's Research Hospital, Memphis, $\mathrm{TN})$ for the HERF $(+)$ and HERF(-) MEL cell clones and for the anti-TRIM10/HERF1 antibody, Dr P Arrigo (CGMC, Université Lyon 1, Villeurbanne, France), Dr P Mehlen (Centre Léon Bérard, Lyon, France) and their collaborators for helping with the MTT and caspase-3/7 assays, Dr H Clevers (Hubrecht Laboratory, Centre for Biomedical Genetics, Utrecht, The Netherlands) for the shRNA vectors, Dr F Moreau-Gachelin and Dr C Guillouf (Inserm U528, Institut Curie, Paris) for the pCDNA6/TR-EF1 $\alpha$ plasmid and the anti-Spi-1/PU.1 antibody and Dr G Mouchiroud and Dr F Morlé (CGMC, Université Lyon 1, Villeurbanne, France) for technical advice and helpful discussions.
This work was supported by grants from the "Ligue contre le Cancer, Comité de la Loire", the "Association de Recherche sur le Cancer" and the "Fondation de France". The authors were supported by the INSERM, the "Ligue contre le Cancer, Comité du Doubs", the "Association de Recherche sur le Cancer" and the "Région RhôneAlpes".

\section{References}

1 Harada H, Harada Y, O'Brien DP, et al. HERF1, a novel hematopoiesis-specific RING finger protein, is required for terminal differentiation of erythroid cells. Mol Cell Biol 1999; 19:38083815.

2 Okuda T, van Deursen J, Hiebert SW, Grosveld G, Downing JR. AML1, the target of multiple chromosomal translocations in human leukemia, is essential for normal fetal liver hematopoiesis. Cell 1996; 84:321-330.

3 Wang Q, Stacy T, Miller JD, et al. The CBFbeta subunit is essential for CBFalpha2 (AML1) function in vivo. Cell 1996; 87:697-708.

4 Meroni G, Diez-Roux G. TRIM/RBCC, a novel class of 'single protein RING finger' E3 ubiquitin ligases. Bioessays 2005; 27:1147-1157.

5 Reymond A, Meroni G, Fantozzi A, et al. The tripartite motif family identifies cell compartments. EMBO J 2001; 20:21402151.

6 Short KM, Cox TC. Subclassification of the RBCC/TRIM superfamily reveals a novel motif necessary for microtubule binding. J Biol Chem 2006; 281:8970-8980.

7 Jensen K, Shiels C, Freemont PS. PML protein isoforms and the RBCC/TRIM motif. Oncogene 2001; 20:7223-7233.

8 Moreau-Gachelin F, Ray D, Mattei MG, Tambourin P, Tavitian A. The putative oncogene Spi-1: murine chromosomal localization and transcriptional activation in murine acute erythroleukemias. Oncogene 1989; 4:1449-1456.

9 Paul R, Schuetze S, Kozak SL, Kozak CA, Kabat D. The Sfpi-1 proviral integration site of Friend erythroleukemia encodes the ets-related transcription factor Pu.1. J Virol 1991; 65:464-467.

10 McKercher SR, Torbett BE, Anderson KL, et al. Targeted disruption of the PU.1 gene results in multiple hematopoietic abnormalities. EMBO J 1996; 15:5647-5658.

11 Scott EW, Fisher RC, Olson MC, et al. PU.1 functions in a cellautonomous manner to control the differentiation of multipotential lymphoid-myeloid progenitors. Immunity 1997; 6:437-447.

12 Anderson KL, Perkin H, Surh CD, et al. Transcription factor PU.1 is necessary for development of thymic and myeloid progenitorderived dendritic cells. J Immunol 2000; 164:1855-1861.

13 Anderson MK, Hernandez-Hoyos G, Diamond RA, Rothenberg EV. Precise developmental regulation of Ets family transcription factors during specification and commitment to the T cell lineage. Development 1999; 126:3131-3148.

14 Guerriero A, Langmuir PB, Spain LM, Scott EW. PU.1 is required for myeloid-derived but not lymphoid-derived dendritic cells. Blood 2000; 95:879-885.

15 Rao G, Rekhtman N, Cheng G, Krasikov T, Skoultchi AI. Deregulated expression of the PU.1 transcription factor blocks murine erythroleukemia cell terminal differentiation. Oncogene 1997; 
14:123-131.

16 Schuetze S, Paul R, Gliniak BC, Kabat D. Role of the PU.1 transcription factor in controlling differentiation of Friend erythroleukemia cells. Mol Cell Biol 1992; 12:2967-2975.

17 Yamada T, Kondoh N, Matsumoto M, et al. Overexpression of PU.1 induces growth and differentiation inhibition and apoptotic cell death in murine erythroleukemia cells. Blood 1997; 89:13831393.

18 Hromas R, Orazi A, Neiman RS, et al. Hematopoietic lineageand stage-restricted expression of the ETS oncogene family member PU.1. Blood 1993; 82:2998-3004.

19 Back J, Dierich A, Bronn C, Kastner P, Chan S. PU.1 determines the self-renewal capacity of erythroid progenitor cells. Blood 2004; 103:3615-3623.

20 Fisher RC, Slayton WB, Chien C, et al. PU.1 supports proliferation of immature erythroid progenitors. Leuk Res 2004; 28:8389.

21 Hallier M, Lerga A, Barnache S, Tavitian A, Moreau-Gachelin F. The transcription factor Spi-1/PU.1 interacts with the potential splicing factor TLS. J Biol Chem 1998; 273:4838-4842.

22 Hallier M, Tavitian A, Moreau-Gachelin F. The transcription factor Spi-1/PU.1 binds RNA and interferes with the RNA-binding protein p54nrb. J Biol Chem 1996; 271:11177-11181.

23 Baklouti F. In: Venables JP, ed. Alternative splicing in cancer. Kerala, India: Transworld Research Network, 2006:235-252.

24 Baklouti F, Huang SC, Tang TK, et al. Asynchronous regulation of splicing events within protein 4.1 pre-mRNA during erythroid differentiation. Blood 1996; 87:3934-3941.

25 Théoleyre O, Deguillien M, Morinière M, et al. Spi-1/PU.1 but not Fli-1 inhibits erythroid-specific alternative splicing of 4.1R pre-mRNA in murine erythroleukemia cells. Oncogene 2004;
23:920-927.

26 Hou VC, Lersch R, Gee SL, et al. Decrease in hnRNP A/B expression during erythropoiesis mediates a pre-mRNA splicing switch. EMBO J 2002; 21:6195-6204.

27 Starck J, Doubeikovski A, Sarrazin S, et al. Spi-1/PU.1 is a positive regulator of the Fli-1 gene involved in inhibition of erythroid differentiation in friend erythroleukemic cell lines. Mol Cell Biol 1999; 19:121-135.

28 van de Wetering M, Oving I, Muncan V, et al. Specific inhibition of gene expression using a stably integrated, inducible smallinterfering-RNA vector. EMBO Rep 2003; 4:609-615.

29 Atar O, Levi BZ. PU.1 silencing leads to terminal differentiation of erythroleukemia cells. Biochem Biophys Res Commun 2005; 329:1288-1292.

30 Lux SE, Palek J. In: Handin RI, Lux SE, Stossel TP, eds. Blood Principles \& Practice of Hematology. Philadelphia: J.B. Lippincott Company, 1995:1701-1818.

31 Chasis JA, Coulombel L, Conboy J, et al. Differentiation-associated switches in protein 4.1 expression. Synthesis of multiple structural isoforms during normal human erythropoiesis. J Clin Invest 1993; 91:329-338.

32 Yergeau DA, Cornell CN, Parker SK, Zhou Y, Detrich III HW. bloodthirsty, an RBCC/TRIM gene required for erythropoiesis in zebrafish. Dev Biol 2005; 283:97-112.

33 Deguillien M, Huang SC, Morinière M, et al. Multiple cis elements regulate an alternative splicing event at $4.1 \mathrm{R}$ pre-mRNA during erythroid differentiation. Blood 2001; 98:3809-3816.

34 Delgado MD, Hallier M, Meneceur P, Tavitian A, Moreau-Gachelin F. Inhibition of Friend cells proliferation by spi-1 antisense oligodeoxynucleotides. Oncogene 1994; 9:1723-1727.

(Supplementary Information is linked to the online version of the paper on the Cell Research website.) 